\title{
Adaptive immunity in invertebrates: a straw house without a mechanistic foundation
}

\author{
Chris Hauton ${ }^{1 *}$ and Valerie J. Smith ${ }^{2}$
}

\begin{abstract}
Summary
Recently claims have been made for radical new insights in the field of invertebrate immunology that involve memory, specificity and/or maternal transfer of immunocompetence. For evidence these claims rely on phenomena, such as survival or reproductive capacity, observed at the level of the whole organism. The allure of these apparently revelatory hypotheses is that they are contrary to established views of innate immunity. They draw implicit analogy to adaptive responses in jawed vertebrates and the terminology used creates an incomplete and misleading picture. We argue that the case for adaptive immunity in invertebrates based only on such phenomena is weak and flawed, as it can only be upheld if supported by descriptions of the underlying mechanisms. We caution against a reliance on this approach as a means of advancing this field-highlighting, as an example, some negative commercial implications of adopting this approach. BioEssays 29:1138-1146, 2007. ๑ 2007 Wiley Periodicals, Inc.
\end{abstract}

\section{Introduction}

Recently observations, made using whole organisms, of invertebrates exposed to experimental infection have been interpreted as evidence that these animals have immunological specificity and 'memory' of a kind that is a 'functional' equivalent of adaptive immunity in gnathostome vertebrates. ${ }^{(1)}$ These observations of phenomena are being claimed as radical new insights into the invertebrate immune system and constitute a major paradigm shift in immunological thinking, rendering current ideas outmoded. These claims for functionally adaptive immunity have been based largely on observations of only a few

\footnotetext{
${ }^{1}$ School of Ocean and Earth Science, University of Southampton, National Oceanography Centre Southampton, UK.

${ }^{2}$ School of Biology, Gatty Marine Laboratory, University of St Andrews, UK.

Funding agencies: The authors would like to acknowledge the support of the UK Biotechnology and Biological Sciences Research Council (BBSRC) and the UK Royal Society.

${ }^{*}$ Correspondence to: Chris Hauton, School of Ocean and Earth Science, University of Southampton, National Oceanography Centre Southampton, European Way, Southampton, Hants SO14 3ZH.

E-mail: ch10@ noc.soton.ac.uk

DOI 10.1002/bies.20650

Published online in Wiley InterScience (www.interscience.wiley.com).
}

species and a limited selection of phenomena. Nonetheless, they have been presented as evidence for innate immune memory or priming, ${ }^{(2-9)}$ innate immune specificity ${ }^{(2,5,8)}$ or the inheritance of immune genotype within invertebrate lineages (i.e. vertical transfer from mother to offspring). ${ }^{(7,8)}$ Hypotheses erected from these observations appear revelatory because the terminology adopted draws analogy to processes that have been characterized in the adaptive immune responses of jawed vertebrates.

In this essay, we argue that a reliance on such phenomenological observations is a flawed and inadequate approach to the study of innate immunity. It sidesteps scientific rigour in failing to rule out, by systematic experimental elimination, other (and arguably simpler) explanations from within the known framework of invertebrate immune functioning. Thus, these hypotheses remain untested and offer no resolution to the problem of elucidating complex host/pathogen systems (see Fig. 1). At best, they are naïve and do little to enhance our understanding of immunology. At worst, they mislead and make a retrograde step back to early research into invertebrate immune systems that was driven by the assumption that invertebrates must possess primitive antibody-type immune responses ancestral to those of vertebrates. Crucially, new 'theories' must take into account known facts about the invertebrate response to non-self agents which have been established for a very wide range of invertebrate taxa and which apply to most invertebrates and most challenges most of the time (Table 1). Put simply, observations of a few 'special cases' of host-parasite interactions in two or three species are not enough to support 'new' global theories, especially without explanation of the biochemical and molecular events involved. We review some of the observational work that has, and is being, used to justify the existence of vertebrate-like adaptive immunity, discuss its limitations and highlight the fundamental necessity for rigorous mechanistic data.

\section{Innate 'priming' or 'memory'}

Phenomenological evidence for 'immune memory ${ }^{\text {(2,9) }}$ or 'immune priming' ${ }^{\text {(3,5-7) }}$ has been used to draw analogies to the repertoire of vertebrate adaptive immune responses. ${ }^{(9)}$ Most reports do not try to make a case for antibody-like molecules or for the clonal selection of specific effectors, but several use a philosophical deductive approach, reasoning 


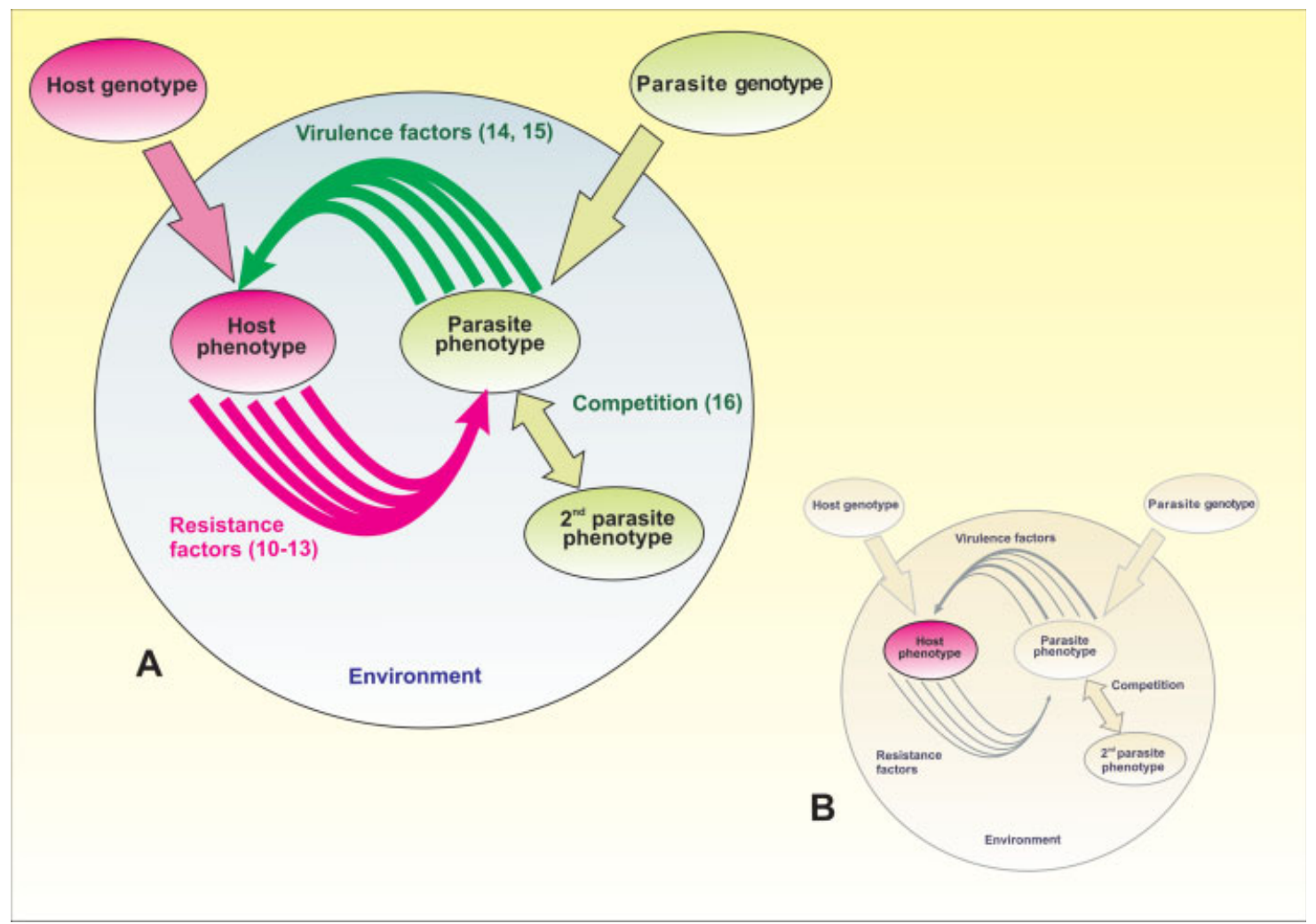

Figure 1. The challenge faced by immune phenomenology. A: A representation of all components of a host-parasite interaction. Host and parasite genotypes are expressed within an environment as phenotypes. Within the environment there are numerous ways in which a host phenotype might counter a parasite phenotype (resistance factors), including behavioural modification, ${ }^{(10)}$ reproductive isolation, ${ }^{(11)}$ a switch in life-history strategy, ${ }^{(12)}$ or immune responses to infection. ${ }^{(13)}$ Similarly there are a number of components which contribute to the virulence of any pathogen including the production of virulence factors, ${ }^{(14)}$ quorum sensing interactions ${ }^{(15)}$ and competition with homologous and heterologous parasites for limited host resources. ${ }^{(16)}$ B: The challenge faced by hypotheses constructed from phenomenological observations of one component of this system (e.g. in this example, host phenotype) is that only one part of a complex whole is investigated, often with little or no consideration of the other components or feedbacks between different components.

that the benefits of acquiring adaptive immunity (i.e. one embracing some degree of specificity and memory) is such that natural selection would have favoured its development. They point out that functionally adaptive immune strategies are as valid as those mediated through vertebrate-type mechanisms and there is no reason why invertebrates should not have invented their own form of 'acquired' or 'adaptive' immunity independently. Whilst these arguments have logic, the existence or not of adaptivity in invertebrates requires: (1) clear, unambiguous and reproducible evidence of at least some specificity and memory that cannot be attributed to anything other than an active response on behalf of the host, (2) a description of the likely mechanism(s) underpinning the response, and (3) extensive experimental testing of these 'new' hypotheses.

The idea that invertebrates have a primitive form of adaptive immune is not new and was explored extensively from the 1960s onwards. Numerous studies were undertaken over some 20 years in which invertebrates were subjected to primary and secondary treatments in a bid to detect elevated immune responsiveness to second or subsequent challenges. Experimental models included graft rejection, phagocytosis, bacterial killing and survival. Despite the prevailing zeitgeist of that time, phenomenological observations and experimental analyses failed to produce consistent and convincing evidence for immune memory or specificity. ${ }^{(30,31)}$ Indeed, it is on the back of this exhaustive and, ultimately, unsuccessful enterprise that comparative immunologists are now confident to assert that invertebrates do not possess vertebrate-like adaptive immunity. ${ }^{(32)}$ The recent papers re-visiting this issue are based more or less entirely on a few individual and unusual host-parasite associations. They cannot therefore offer a general new concept that can be applied across a range of invertebrate taxa. 


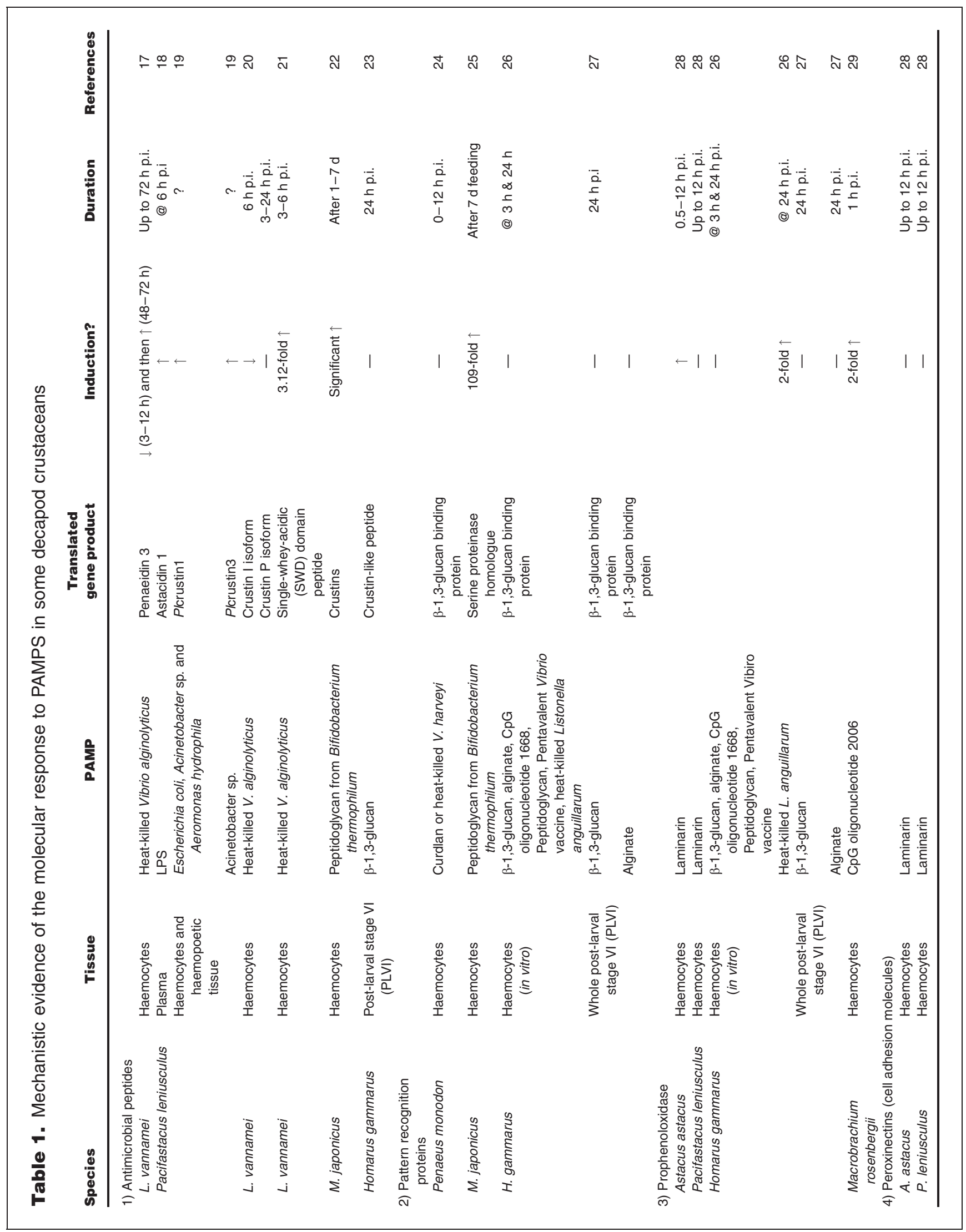


Some proponents of immune priming and memory base their case on experiments in which pre-exposure to particular challenges, particularly lipopolysaccharides or $\beta$-1,3-glucans, are used to prepare the immune system for subsequent pathogen challenge. Lipopolysaccharides and glucans are compounds known to immunologists as pathogenassociated molecular patterns (PAMPs) as they are derived from bacterial or fungal pathogens, respectively. They bind to molecules within the host immune system, the pattern recognition receptors (PRRs), to form a complex that then initiates a series of events, such as signal transduction or activation of enzyme cascades that result in the liberation of bioactive molecules. What actually happens when protostome invertebrates are injected with PAMPs is that a full-blown immune response occurs, involving activation of particular enzyme cascades, such as the prophenoloxidase system, the regulated exocytosis of aggressive molecules, ${ }^{(4,13)}$ an increase in the expression of genes coding for antimicrobial proteins $^{(17,20,23)}$ and /or other related proteins (see Table 1 and Smith et al. $\left.{ }^{(33)}\right)$, and the initiation of encapsulation reactions leading to haemocytopenia. Experiments in which presentation of PAMPs to the protostome immune system has been claimed to 'prime' the immune system, measure not immune parameters, as might be expected, but survival, ${ }^{(5)}$ temporary increases in growth ${ }^{(34,35)}$ or reproductive capacity. ${ }^{(8,36)}$ Thus it is peremptory to then invoke 'adaptivity' by way of explanation for any positive outcomes observed.

Importantly, all responses induced by experimental 'priming' have temporal signatures, such as the activation or release of immune system components, which continue until all PAMPs are cleared. In the case of a successful immune response, the released and activated components will persist within the haemocoel until broken down by the host or, in the case of degranulated haemocytes, are replaced by recruitment of haemocytes to the site of infection ${ }^{(17)}$ and haemopoiesis. ${ }^{(37)}$ In decapod crustaceans, mechanistic evidence of this temporal signature has definitively been shown to last between 3 and 72 hours (reviewed by Smith et al., ${ }^{(33)}$ see also Table 1). Thus any effect on the immune system is short-lived and the 'cost' to the host is potentially high. Inappropriate 'priming' will ultimately deplete the immune system of its chemical and cellular components causing it to become exhausted. If 'priming' does indeed improve survival, growth or fecundity, something else is happening that doesn't necessarily require the creation of an 'adaptive' immune response that has been overlooked previously. In many cases, the experimental design could be the factor that influences the result, not the presence of immune memory.

To our knowledge, there has been only one recent report of priming and 'memory' that has attempted to identify the underlying mechanism involved, and this is the specific case in a Drosophila-Streptococcus pneumoniae host-pathogen model. ${ }^{(5)}$ In this laboratory model, it is argued that exposure to a sublethal dose of $S$. pneumoniae 7 days before a lethal dose of the same species of bacteria does not stimulate the transcription of antimicrobial peptides or the activation of the prophenoloxidase (proPO) enzyme system, but nonetheless prolongs survival of the host. In this specific example, which is not repeated in other Drosophila-bacterial pathogen models, the published data indicate that the phagocytes are responsible for subsequent immunity and that their activity is primed by the experience of the initial sublethal dose. However, it is not clear from these data whether this activity is attributable to an increased performance of existing phagocytes, to an increase in the overall number of phagocytes in vivo, or to some other aspect of the immune system that has not been characterised in the study but which is important to this artificial hostpathogen system (for example: the stimulation of oxyradical production or of proPO-related immune system components that cannot be monitored from gross assessments of melanisation). As a consequence, this publication, ${ }^{(5)}$ whilst going further than most others to investigate the possible mechanism(s) underpinning the observed response, in fact highlights the challenge of dissecting complex immune processes, especially from limited phenomenological observations recorded in isolation. The available evidence does not exclude the possibility that other immune system components are stimulated and does not offer sufficient evidence to justify regarding the response as a newly discovered example of 'immune priming' or memory. We believe that such a conclusion can be reached only after all known immune processes in the host have been tested and eliminated by direct experimental methods.

Moreover, the descriptor 'immune memory' is not appropriate as it implicitly draws analogy to the vertebrate-acquired response, which is functionally entirely different, relying on clonally derived lymphocyte subsets, RAG genes and MHC I and II, ${ }^{(32)}$ all of which are the key mediators and hallmarks of immune memory in mammals and none of which appear, to date, to exist in invertebrates, urochordates or agnathans. In no sense does the temporary persistence of immune system components within the haemocoel compare with the life-long production of specific immune cells or antibodies, which typify the immune memory of jawed-vertebrates. Consequently similar terminologies should be avoided.

\section{Innate specificity}

Many of the arguments made in favour of invertebrate immune memory based on phenomenological observations claim that it also exhibits specificity. ${ }^{(2,5,6,8,9)}$ Re-infection level is the measured parameter in a study of the copepod, Macrocyclops albidus, and its parasite, Schistocephalus solidus. ${ }^{(2)}$ The authors report lower levels of infection in the host after prior exposure to the parasite. As with the Drosophila-S. pneumoniae model, discussed above, ${ }^{(5)}$ the response seems to be 'specific' because it is not seen if the second challenge is 
to a different 'strain' of the same parasite. ${ }^{(2)}$ Precisely what constitutes the genotypic or antigenic differences between the strains of parasites is not defined yet the authors interpret their findings as evidence for specific memory in the copepod immune system. ${ }^{(2)}$ Furthermore, no direct measurements were made of immune parameters or of parasite killing and the effect was studied over only three days. No mechanistic evidence for this apparent specificity has been presented and no framework for experimental validation so far has been put forth.

Arguing against specificity in the invertebrate immune system is the abundant mechanistic data that have established that the innate immune response of invertebrates is rather non-specific. For example, it has been shown that inoculation with $\beta$-1,3-glucans, derived from fungal cell walls, increases the antibacterial response of Bombus terrestris to Arthrobacter globiformis. ${ }^{(38)}$ Numerous other workers have also reported non-specific responses to a diverse array of putative PAMPs including yeast extracts, alginates, sulfated polysaccharides and so forth. ${ }^{(39-43)}$ With respect to the Drosophila/Streptococcus system described above, no matter how intriguing the data are, this single case does not support a general theory of the evolution of specificity in innate immunity.

Whilst existing molecular and cellular data indicate that invertebrates possess innate immune capability with nonspecific responses, it does not follow that they are only able to respond to a limited range of non-self entities. On the contrary, invertebrates are able to respond to a remarkably wide diversity of non-self moieties. The genome size of invertebrates is too small for them to encode suites of receptors to the hundreds of thousands of non-self agents that could potentially threaten the homeostatic integrity of the host ${ }^{(44)}$ and it is the holy grail of immunologists to discover how invertebrate systems manage to achieve such an astounding capacity for recognition. No better case for the absolute importance of mechanistic study at a cellular and molecular level could be made than by recent studies of diversity in the immune systems of the sea lamprey Petromyzon marinus, ${ }^{(45)}$ the sea urchin Strongylocentrotus purpuratus, ${ }^{(46)}$ the gastropod Biomphalaria glabrata ${ }^{(4)}$ and the mosquito Anopheles gambiae. ${ }^{(48)}$ Molecular studies in $A$. gambiae have shown that the AgDscam gene can undergo alternative exon splicing to produce a repertoire of pattern recognition receptors (PRRs) on the surface of circulating haemocytes. ${ }^{(48)}$ In B. glabrata an alternative mechanism appears to have evolved in that an extensive family of fibrinogen-related genes (FREP genes) with lectin-like properties have been identified. The expression of these genes has been shown to be upregulated after infection with digenean parasites such as Echinostoma paraensei. $^{(47)}$ Expressed sequence tag (EST) studies in $S$. purpuratus have shown that the expression of the large 185/333 gene family is increased after inoculation with lipopolysaccharide. ${ }^{(46)}$ However, the ability to somatically expand receptor diversity does not, on its own, constitute memory or effector specificity as recently put forward. ${ }^{(49)}$ To make such conceptual leaps to support the case for vertebrate style adaptive immunity in invertebrates without any other basis is little short of mechanistic justification by proxy.

\section{Further limitations of phenomenology-an incomplete picture of inheritance}

The failure to make assessment of immunological parameters is a consistent weakness in most papers purporting to demonstrate priming, memory or adaptivity in the invertebrate innate immune system. In some cases, only single-end points are taken and the role of the immune system is inferred but not actually tested. One example is Daphnia magna and its obligate endoparasite, Pasteuria ramosa, ${ }^{(8)}$ a bacterium that infests the body cavity of its host leading to early reproduction, gigantism and, eventually, castration. ${ }^{(50)}$ The authors report that prior exposure of $D$. magna to one strain of $P$. ramosa enables the first three parthenogenically produced clutches of offspring to be more resistant to challenge by the same strain but not a different one. ${ }^{(8)}$ They contend that this provides evidence of maternally transferred strain-specific acquired immunity and their paper is frequently cited in support of the existence of immune memory in invertebrates. However, 'resistance' of the host in this investigation is measured in terms of the fecundity (number of offspring and time to first clutch) of the daughter clones. There is no measurement of any immune parameters in the hosts. So how can these observations actually be explained in terms of immune reactivity? It might be expected that the host immune response would act to kill or eliminate the parasites but whether or not the Daphnia daughter clones of the prior challenged mothers had reduced the parasite loads of $P$. ramosa compared to unchallenged controls is not made clear. A later paper has reported that treatment of infected $D$. magna with antibiotics ' cures' infection and reverses castration, ${ }^{(50)}$ so a more reliable measure of in this case might also be reduced infection and castration rates. Curiously though, it appears that infected Daphnia actually have enhanced reproduction prior to castration and host death, the time when the new parasites are shed as spores. ${ }^{(50)}$

Ultimately, however, changes in reproductive output cannot be taken as evidence of immune response; at best it can be seen as evidence for a shift in the life history strategy of $D$. pulex, as in fact reported in a later study of fecundity compensation in the D. pulex/Glugoides intestinalis host/ parasite system. $^{(12)}$ Fecundity compensation is a wellestablished principle in the field of parasitology ${ }^{(51,52)}$ where it has been argued to be an energetically conservative evolutionary solution to the problem presented by the hostparasite evolutionary 'arms race'. There would be some advantage to the mothers as earlier reproduction gives her offspring a small lead over uninfected con-specifics in gaining

\section{BioEssays 29.11}


first access to resources and enabling them to live long enough to reproduce themselves, irrespective of the risk of later infection. Thus one would not expect to see the evolution of acquired host resistance; a subsequent laboratory study ${ }^{(53)}$ seems to uphold this. Indeed, a later paper has shown for Daphnia that the young of females subjected to poor environmental conditions (food supply and water quality) seem less susceptible to $P$. ramosa infection than those produced by females in a good environment. ${ }^{(54)}$ This does not demonstrate maternal transfer of specific immunity against this parasite. A more parsimonious explanation is that the mothers raised in a poor environment produce fewer young, earlier, in a bid for survival, perhaps favouring those embryos genotypically best 'fitted' to deal with any infection. Unfortunately no information was provided about clutch size between the environmentally disadvantaged mothers and those from better conditions. Fairly routine molecular analyses could also have revealed genotypic and phenotypic differences between the experimental and control embryos.

Certainly, recent molecular immune studies ${ }^{(27)}$ (see also Fig. 2) have begun to provide tentative evidence for invertebrates that some individuals might be genetically better able to respond to non-self challenges, indicating that it may be possible that generational enrichment of immune genotypes/phenotypes in the population could occur in sexually reproductive invertebrates, although not automatically requiring transfer of specific immunity. In the case of decapods, specifically lobster (Homarus gammarus) post-larvae, we have found that a small but distinct proportion of animals show higher levels of immune gene expression than others, irrespective of whether the post-larvae had been pre-treated with PAMP-containing agents or not. In short, an animal expressing one immune gene highly is likely to express other immune genes highly as well. ${ }^{(27)}$ These preliminary findings have yet to be substantiated with a wider range of immuneassociated and non-related genes, but it is known within shrimp farming that selective breeding can be used to produce shrimp phenotypes better able to withstand specific diseases, such as the Taura syndrome virus. ${ }^{(55)}$ Fundamental mechanistic studies on the genetics of immune traits are now essential to clarify our understanding of the nature of the invertebrate immune system and the role, if any, of inheritance.

\section{Further limitations-ignoring the pathogens}

Hypotheses based on observations of host phenomena can also completely ignore the role of the parasite in the interaction. Indeed, the importance of the parasites' role has recently been emphasized by data presented ${ }^{(56)}$ from the $D$. magna/P. ramosa system which identified the optimal model for parasite virulence as that in which the castrating parasite maximizes lifetime transmission success by converting host reproductive resources into transmission stages. Similarly interstrain parasite competition within a host (see

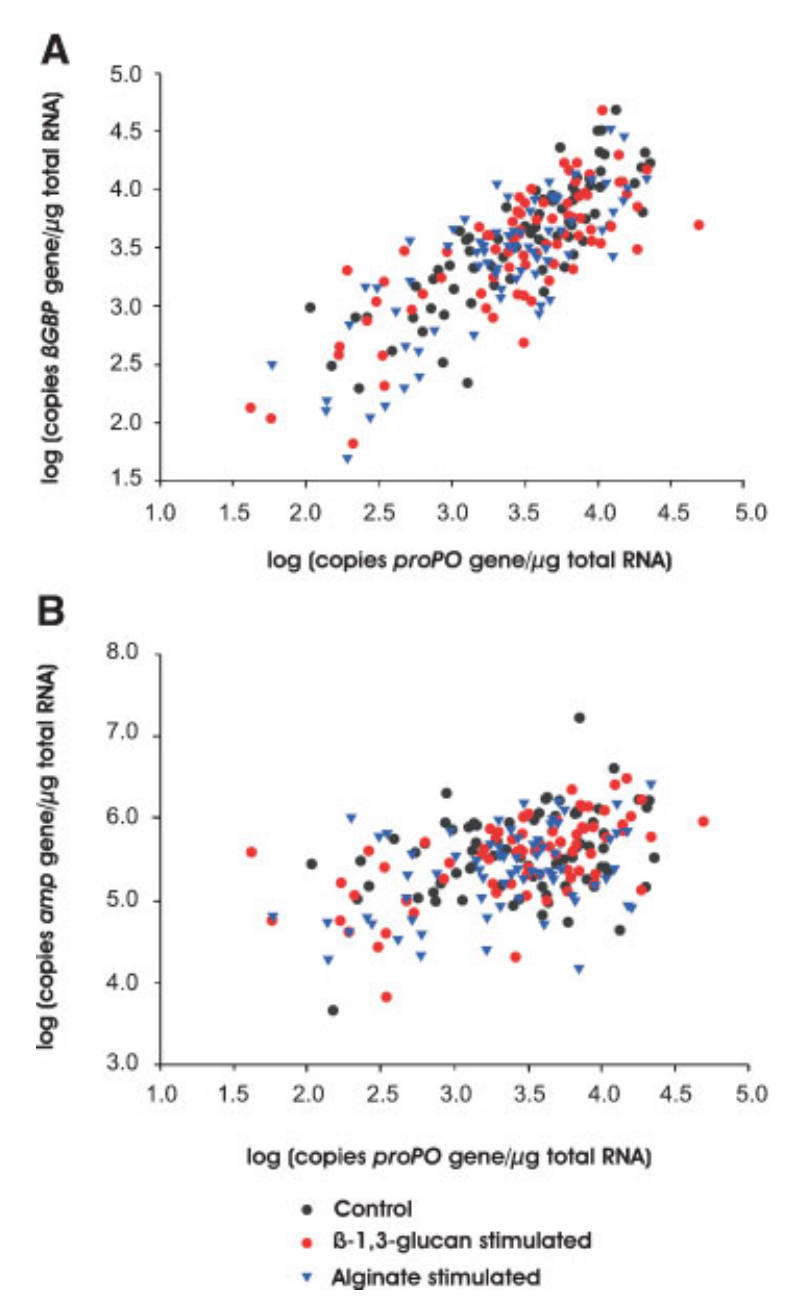

Figure 2. Mechanistic evidence for immune inheritance? Significant $(P<0.001)$ correlations between the expression of genes encoding the lobster, $H$. gammarus, prophenoloxidase enzyme (proPO gene) and the $\beta-1,3$-glucan binding protein ( $\beta G B P$ gene) or a putative antimicrobial peptide (amp gene). The figure shows that, irrespective of whether the lobster postlarvae received an immune stimulant, a subset of each population expressed high numbers of transcripts for all genes whilst another subset expressed low numbers of mRNA transcripts for each gene. This indicates that immune expression was determined by some other factor, possibly genotypic inheritance from the parents (reproduced with permission from: Molecular Immunology 44, Hauton C, Brockton V \& Smith VJ. In vivo effects of immunostimulants on gene expression and disease resistance in lobster (Homarus gammarus) post-larval stage VI (PLVI) juveniles, 443-450, Copyright Elsevier (2007)).

Fig. 1) must be considered. Competition for resources (including a host) may be expected to be more intense between more closely related parasite strains with similar resource requirements. Intense competition between highly related parasites, and vice versa, could explain the gradation 
of response often reported in observational studies. ${ }^{(2,8)}$ Competition between parasites has also been identified in several viral diseases of crustaceans. Tang and co-authors ${ }^{(16)}$ demonstrated that the survival of the blue shrimp, Litopenaeus stylirostris, after infection with White Spot Syndrome Virus (WSSV) can be improved by prior infection with Infectious Hypodermal and Haemopoetic Necrosis Virus (IHHNV). These authors argue that the negative interaction of the two viruses, a process referred to as viral interference, ${ }^{(57)}$ in which both viruses compete for the limited replication resources of host cell, causes this situation. Clearly without a mechanistic understanding of both host-parasite and parasite-parasite interactions it is difficult, if not impossible, to attribute 'immune' phenomena to host immune function alone.

\section{The commercial danger of phenomenology-shellfish aquaculture}

These considerations have a practical importance and do not represent simply theoretical semantic arguments. The shellfish aquaculture industry is desperate to find ways to minimize the consequences of disease outbreaks, and is seeking to develop prophylactics to protect stock animals from infection. (33) Phenomenological observations of 'priming', functional adaptivity or 'memory' continue to provide the 'scientific basis' to support the aquaculture pharmaceutical industry. ${ }^{(58-60)}$ Numerous products are now marketed globally as additives to enhance the immunity of commercially cultured species, mainly shrimp. As the shellfish farming industry continues to grow, successful treatments that acquire good 'credibility' are set to become 'big earners' for the manufacturers. As previously reviewed ${ }^{(33)}$ however, all mechanistic studies so far have failed to support the conclusion that any stimulation of the invertebrate immune system is sufficiently long-lived to constitute a proper solution to the problem of recurrent disease within the context of invertebrate aquaculture. The limits of immune stimulation as a strategy have only been realized with the advent of detailed studies of cellular, protein and molecular processes, questioning the reliance on phenomena in isolation as a means of advancing or exploiting our knowledge of invertebrate innate immune systems.

\section{Conclusion}

In this review, we argue that there is insufficient unequivocal information to support the case for adaptive immunity in invertebrates, at least of the type shown by the gnathostomes. We do not wish to discredit or discourage attempts to understand invertebrate immunity from evolutionary, ecological or phenomenological standpoints. In fact, we fully acknowledge that phenomenological investigations are a logical starting point and immunology is as informed by evolutionary theory as much as any biological discipline. However, we believe that observation cannot be used in isolation and should not be used solely as the basis for radical claims that run counter to well-established mechanistic evidence. They can, of course, be used to construct hypotheses but such hypotheses must be exhaustively tested and backed by rigorous functional cellular, biochemical and molecular methods, to eliminate all alternative explanations and existing data even if thought 'old' should not be ignored (Table 1, see also $^{(51,52,57)}$ ).

Importantly, it is necessary to establish if any mechanisms found are homolgous to those in vertebrates. This last point is crucial because as Klein ${ }^{(44)}$ has cogently pointed out, functional similarity in the immune system does not prove homology and is a meaningless concept.

We do not need to re-ask questions addressed and answered 30-40 years ago and hope to find that after all we have been missing something obvious all this time. Many, if not all, of the phenomena currently purported to show adaptive responses in invertebrates can be explained within the framework of our current knowledge of the multitude of innate processes. Only when we are sure that they cannot, do we have something truly radical to celebrate.

\section{Acknowledgments}

We acknowledge the assistance of two anonymous referees and the editor in revising the manuscript.

\section{References}

1. Little TJ, Hultmark D, Read AF. 2005. Invertebrate immunity and the limits of mechanistic immunology. Nat Immunol 6:651-654.

2. Kurtz J, Franz K. 2003. Evidence for memory in invertebrate immunity. Nature 425:37-38

3. Little TJ, Kraaijeveld AR. 2004. Ecological and evolutionary implications of immunological priming in invertebrates. Trend Ecol Evol 19:58-60.

4. Moret Y, Siva-Jothy MT. 2003. Adaptive innate immunity? Responsivemode prophylaxis in the mealworm beetle, Tenebrio molitor. Proc R Soc Lond B 270:2475-2480

5. Pham LN, Dionne MS, Shirasu-Hiza M, Schneider DS. 2007. A specific primed immune response in Drosophila is dependent on phagocytes. PLOS Path 3:e26.

6. Schmid-Hempel P. 2005. Natural insect host-parasite systems show immune priming and specificity: puzzles to be solved. BioEssays 27: 1026-1034.

7. Sadd BM, Kleinlogel Y, Schmid-Hempel R, Schmid-Hempel P. 2005. Trans-generational immune priming in a social insect. Biol Lett 1:386388.

8. Little TJ, O'Connor B, Colegrave N, Watt K, Read AF. 2003. Maternal transfer of strain-specific immunity in an invertebrate. Curr Opin Biol 13:489-492.

9. Kurtz J. 2005. Specific memory within innate immune systems. Trends Immunol 26:186-192.

10. Behringer DC, Butler MJ, Shields JD. 2006. Avoidance of disease by social lobsters. Nature 441:421.

11. Jaenike J, Dyer KA, Cornish C, Minhas MS. 2006. Asymmetrical reinforcement in Wolbachia infection in Drosophila. PLOS Biol 4:1852-1862.

12. Chadwick W, Little TJ. 2005. A parasite-mediated life-history shift in Daphnia magna. Proc R Soc B 272:505-509.

13. Smith VJ, Söderhäll K. 1983. Induction of degranulation and lysis of haemocytes in the freshwater crayfish, Astacus astacus by components of the prophenoloxidase activating system in vitro. Cell Tiss Res 233: 295-303.

14. Dzikowski R, Frank M, Deitsch K. 2006. Mutually exclusive expression of virulence genes by malaria parasites is regulate independently of antigen production. PLOS Path 2:184-194.

\section{BioEssays 29.11}


15. Defoirdt T, Bossier P, Sorgeloos P, Verstraete W. 2005. The impact of mutations in the quorum sensing systems of Aeromonas hydrophila, Vibrio anguillarum and Vibrio harveyi on their virulence to gnotobiotically cultured Artemia franciscana. Environ Microbiol 7:1239-1247.

16. Tang KFJ, Durand SV, White BL, Redman RM, Mohney LL, et al 2003. Induced resistance to white spot syndrome virus infection in Penaeus stylirostris through pre-infection with infectious hypodermal and hematopoietic necrosis virus-a preliminary study. Aquacult 216 19-29.

17. Muñoz M, Vandenbulcke F, Saulnier D, Bachère E. 2002. Expression and distribution of penaeidin antimicrobial peptides are regulated by haemocyte reactions in microbial challenged shrimp. Eur $\mathrm{J}$ Biochem 269:2678-2689

18. Lee SY, Lee BL, Söderhäll K. 2003. Processing of an antibacteria peptide from hemocyanin of the freshwater crayfish Pacifastacus lenisuculus. J Biol Chem 278:7927-7933.

19. Jiravanichpaisal $P$, Lee SY, Kim Y-A, Andrén T, Söderhäll I. 2007 Antibacterial peptides in haemocytes and hematopoietic tissue from freshwater crayfish Pacifastacus leniusculus: characterization and expression pattern. Dev Comp Immunol 31:441-455.

20. Vargas-Albores F, Yepiz-Plascencia G, Jiménez-Vega F, Ávila-Villa A 2004. Structural and functional differences of Litopenaeus vannamei crustins. Comp Biochem Physiol B 138:415-422.

21. Jiménez-Vega J, Yepiz-Plascencia G, Söderhäll K, Vargas-Albores F 2004. A single WAP domain-containing protein from Litopenaeus vannamei haemocytes. Biochem Biophys Res Comm 314:681687

22. Rattanachai A, Hirono I, Ohira T, Takahashi Y, Aoki T. 2004. Cloning of kuruma prawn Marsupenaeus japonicus crustin-like peptide cDNA and analysis of its expression. Fish Sci 70:765-771.

23. Hauton C, Brockton V, Smith VJ. 2006. Cloning of a crustin-like, single whey-acidic-domain, antibacterial peptide from the haemocytes of the European lobster, Homarus gammarus, and its response to infection with bacteria. Mol Immunol 43:1490-1496.

24. Sritunyalucksana K, Young Lee S, Söderhäll K. 2002. A $\beta-1,3-g l u c a n$ binding protein from the black tiger shrimp Penaeus monodon. Dev Comp Immunol 26:237-245.

25. Rattanachai A, Hirono I, Ohira T, Takahashi Y, Aoki T. 2005 Peptidoglycan inducible expression of a serine proteinase homologue from Kuruma shrimp (Marsupenaeus japonicus). Fish Shell Immunol 18 39-48.

26. Hauton C, Hammond JA, Smith VJ. 2005. Real-time PCR quantification of the in vitro effects of crustacean immunostimulants on gene expression in lobster (Homarus gammarus) granular haemocytes. Dev Comp Immunol 29:33-42.

27. Hauton C, Brockton V, Smith VJ. 2007. In vivo effects of immunostimulants on gene expression and disease resistance in lobster (Homarus gammarus) post-larval stage VI (PLVI) juveniles. Mol Immunol 44:443450

28. Cerenius L, Bangyeekhun E, Keyser P, Söderhäll I, Söderhäll K. 2003. Host prophenoloxidase expression in freshwater crayfish is linked to increased resistance to the crayfish plague fungus, Aphanomyces astaci. Cell Microbiol 5:353-357.

29. Lu K-Y, Huang Y-T, Lee H-H, Sung H-H. 2006. Cloning the prophenoloxidase cDNA and monitoring the expression of proPO mRNA in prawns (Macrobrachium rosenbergii) stimulated in vivo by CpG oligonucleotides. Fish Shell Immunol 20:274-284.

30. Cooper E. 1974. Contemporary Topics in Immunobiology, Vol. 4 London: Plenum Press.

31. Maramorosch K, Shope RE. 1975. Invertebrate Immunology. London: Academic Press.

32. Flajnik MF, Du Pasquier L. 2004. Evolution of innate and adaptive immunity: can we draw the line? Trends Immunol 25:640-644.

33. Smith VJ, Brown JH, Hauton C. 2003. Immunostimulation in crustaceans: does it protect against infection? Fish Shell Immunol 15 $71-90$.

34. Pan CT. 1965. Studies on the host-parasite relationship between Schistosoma mansoni and the snail Australorbis glabratus. Am J Trop Med Hyg 14:931-976.
35. Loker ES. 1979. Effects of Schistosomatium douthitti infection on the growth, survival and reproduction of Lymnaea stagnalis. Exp Parasitol 24:137-146.

36. Minchella DJ, Loverde PT. 1981. A cost of increased early reproductive effort in the snail Biomphalaria glabrata. Am Nat 118: 876-881.

37. Söderhäll I, Bangyeekhun E, Mayo S, Söderhäll K. 2003. Hemocyte production and maturation in an invertebrate animal; proliferation and gene expression in hematopoietic stem cells of Pacifastacus leniusculus. Dev Comp Immunol 27:661-672.

38. Korner P, Schmid-Hempel P. 2004. In vivo dynamics of an immune response in the bumble bee Bombus terrestris. J Invert Pathol 87: 59-66.

39. Sung H-H, Kou GH, Song YL. 1994. Vibrosis resistance induced by glucan treatment in tiger shrimp (Penaeus monodon). Fish Pathol 29: $11-17$.

40. Takahashi $\mathrm{Y}$, Kondo $\mathrm{M}$, Itami $\mathrm{T}$, Honda $\mathrm{T}$, Inagawa $\mathrm{H}$, et al. 2000. Enhancement of disease resistance against penaeid acute viraemia and induction of virus-inactivating in haemolymph of kuruma shrimp, Penaeus japonicus, by administration of Pantoea agglomerans lipopolysaccharide (LPS). Fish Shell Immunol 10:555558.

41. Soto SA, Viana MT. 2003. Effect of sulfated carrageenan on the cellular response of the lobster Panulirus interruptus. Ciencias marinas 29:155168

42. Burgents JE, Burnett KG, Burnett LE. 2004. Disease resistance of the Pacific white shrimp, Litopenaeus vannamei, following the dietary administration of a yeast culture food supplement. Aquacult 231:1-8.

43. Montero-Rocha A, Mclntosh D, Sánchez-Merino R, Flores I. 2006. Immunostimulation of white shrimp (Litopenaeus vannamei) following dietary administration of Ergosan. J Invert Pathol 91:188194.

44. Klein J. 1997. Homology between immune responses in vertebrates and invertebrates: Does it exist? Scand J Immunol 46:558564.

45. Pancer Z, Amemiya CT, Ehrhardt GRA, Ceitlin J, Gartland GL, et al. 2004. Somatic diversification of variable lymphocyte receptors in the agnathan sea lamprey. Nature 430:174-180.

46. Terwilliger DP, Buckley KM, Mehta D, Moorjani PG, Smith LC. 2006. Unexpected diversity displayed in cDNAs expressed by the immune cells of the purple sea urchin, Strongylocentrotus purpuratus. Physiol Genomics 26:134-144

47. Léonard PM, Adema CM, Zhang S-M, Loker ES. 2001 Structure of two FREP genes that combine IgSF and fibrinogen domains, with comments on the diversity of the FREP gene family in the snail Biomphalaria glabrata. Gene 269:155-165

48. Dong Y, Taylor HE, Dimopoulos G. 2006. AgDscam, a hypervariable immunoglobulin domain-containing receptor of the Anopheles gambiae innate immune system. PLOS Biol 4:e229.

49. Kurtz J, Armitage SAO. 2006. Alternative adaptive immunity in invertebrates. Trends Immunol 27:493-496.

50. Ebert D, Carius HJ, Little TJ, Decaestecker E. The evolution of virulence when parasites cause host castration and gigantism. Am Nat 164:S19 S32.

51. Minchella DJ. 1985. Host life-history variation in response to parasitism Parasitol 90:205-216.

52. Forbes MRL. 1993. Parasitism and host reproductive effort. Oikos 67:444-450.

53. Mitchell SE, Read AF, Little TJ. 2004. The effect of a pathogen epidemic on the genetic structure and reproductive strategy of the crustacean Daphnia magna. Ecol Lett 7:848-858.

54. Mitchell SE, Read AF. 2005. Poor maternal environment enhances offspring disease resistance in an invertebrate. Proc R Soc B 272:26012607

55. Argue BJ, Arce SM, Lotz JM. Moss SM. 2002. Selective breeding of Pacific white shrimp (Litopenaeus vannamel) for growth and resistance to Taura syndrome virus. Aquacult 204:447460. 
56. Jensen KH, Little TJ, Skorping A, Ebert D. 2006. Empirical support for optimal virulence in a castrating parasite. PLOS Biol 4:1265-1269.

57. Fenner F, McAuslan BR, Mims CA, Sambrook J, White DO. 1974. The biology of animal viruses, 2nd edition. New York NY: Academic Press. $834 \mathrm{p}$

58. Keith IR, Paterson WD, Aidrie D, Boston LD. 1992. Defence mechanisms of the American lobster (Homarus americanus): vaccination provided protection against gaffkemia infections in laboratory and field trials. Fish Shell Immunol 2:109-119.
59. Boonyaratpalin S, Boonyaratpalin M, Supamattaya K, Toride Y. 1995. Effects of peptidoglycan (PG) on growth, survival, immune response, and tolerance to stress in black tiger shrimp, Penaeus monodon. In: Shariff $M$, Arthur JR, Subasinghe $P$, editors. Diseases in Asian Aquaculture II. Fish Health Section. Manila, Philippines: Asian Fisheries Society. pp 469-477.

60. Song L, Liu JJ, Chan LC, Sung HH. 1997. Glucan induced disease resistance in tiger shrimp (Penaeus monodon). Dev Biol Standard 90: 413-421.

\section{BioEssays 29.11}

